\title{
On the Self-Generation of Electrical Soliton Pulses
}

\author{
David S. Ricketts, Member, IEEE, Xiaofeng Li, Student Member, IEEE, Nan Sun, Student Member, IEEE,
} Kyoungho Woo, Student Member, IEEE, and Donhee Ham, Member, IEEE

\begin{abstract}
The nonlinear transmission line is a structure where short-duration pulses called electrical solitons can be created and propagated. By combining, in a closed-loop topology, the nonlinear line and a special amplifier that provides not only gain but also mechanisms to tame inherently unruly soliton dynamics, we recently constructed the first electrical soliton oscillator that self-generates a stable, periodic train of electrical soliton pulses (Ricketts et al., IEEE Trans. MTT, 2006). This paper starts with a review of this recently introduced circuit concept, and then reports on new contributions, i.e., further experimental studies of the dynamics of the stable soliton oscillator and a CMOS prototype demonstrating the chip-scale operation of the stable soliton oscillator. Finally, we go to the opposite end of the spectrum and present a numerical study showing the possibilities that deliberate promotions of the unruly soliton dynamics in the closed-loop topology can produce chaotic signals.
\end{abstract}

Index Terms-Electrical soliton oscillators, electrical solitons, chaos, integrated circuits, mode-locking, nonlinear transmission lines, oscillators, pulse generation, solitons.

\section{INTRODUCTION}

$\mathbf{S}$ HORT-DURATION electrical pulses play important roles in ultrafast time-domain metrology: they are used to sample rapidly varying signals or as probe signals in ranging radars and time-domain reflectometry [2]-[4]. In addition, possible use of short pulses as career signals in communication has been actively investigated [5].

The nonlinear transmission line (NLTL), a 1-D lattice of inductors and varactors [Fig. 1(a)], stands as one of the most powerful vehicles to generate short electrical pulses [6]. This is due to NLTL's unique ability to shape an input signal into a sharp, spatially localized pulse known as a soliton: Fig. 1(a) shows an example where an input pulse is compressed into a soliton. The NLTL has been extensively studied over the past 40 years [6]-[8], culminating in a monolithic structure that can achieve a pulse rise time as low as 480 fs [8]. In these past 40 years of works, however, the NLTL has been used almost exclusively as a two-port (input + output) device that requires a high-frequency input to produce a soliton pulse output [Fig. 1(a)].

Manuscript received December 7, 2006; revised March 24, 2007. This work was supported by the National Science Foundation (NSF) under Grant ECS0313143 and the Army Research Office (ARO) under Grant W911NF-06-10290.

D. S. Ricketts is with the Department of Electrical and Computer Engineering, Carnegie Mellon University, Pittsburgh, PA 15232 USA (e-mail: ricketts@ece.cmu.edu).

X. Li, N. Sun, K. Woo, and D. Ham are with Electrical Engineering, School of Engineering and Applied Sciences, Harvard University, Cambridge, MA 02138 USA (e-mail: donhee@ seas.harvard.edu).

Digital Object Identifier 10.1109/JSSC.2007.900291

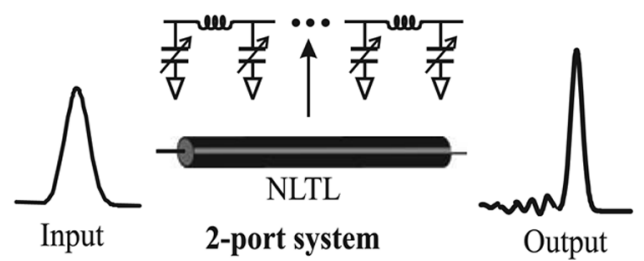

(a)

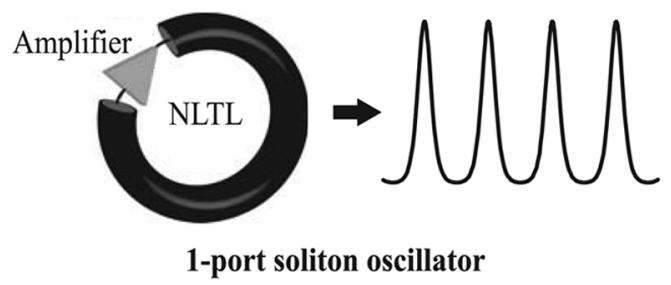

(b)

Fig. 1. (a) Two-port NLTL. (b) One-port electrical soliton oscillator.

Reporting in [1], the authors recently introduced the first one-port (output-only) electrical circuit that robustly self-generates a periodic, stable train of electrical soliton pulses with no high-frequency input. This electrical soliton oscillator, as an autonomous and self-contained (no input needed) source of electrical solitons, marks a distinctive departure from the traditional two-port NLTL.

Our soliton oscillator [1] was made possible by combining an NLTL with a special amplifier in a circular topology [Fig. 1(b)]. By nature, solitons circulating in such an oscillatory loop are "unruly," i.e., they tend towards significant amplitude and phase variations as a result of continual collisions amongst co-circulating solitons. This oscillation instability had been previously difficult to address [1]. The key to our success in building the stable soliton oscillator was finding a way to "tame" the unruly solitons and incorporating the taming mechanism in the amplifier. In optics, lasers that self-generate a train of light wave solitons are a well-known technology [9]. Our soliton oscillator may be understood as an electrical analogue of such soliton lasers.

The present paper consists of two thematic parts. The first part is a review on the following topics:

- Section II: NLTL and electrical solitons.

- Section III: Our soliton oscillator work to date [1] (this work was also reviewed in [10]).

This review part is prepared to share the still-fresh soliton oscillator concept with this journal readership and to facilitate the reading of the rest of the paper.

The second part reports on new contributions from our continuing soliton oscillator research. It is broken down as follows:

- Section IV: Further experimental studies on the soliton oscillator dynamics using the discrete prototype of [1]. 
(a)

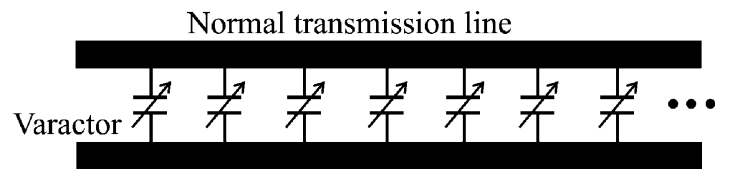

(b)

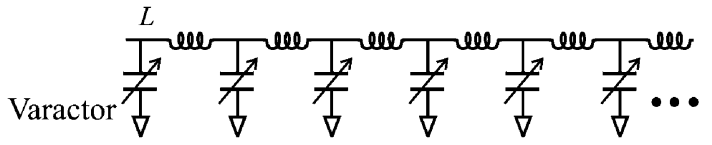

(c)

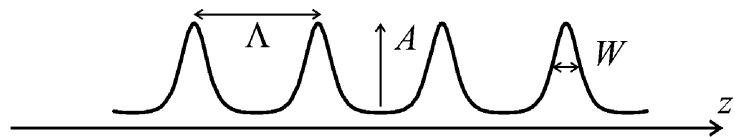

Fig. 2. (a) An NLTL. (b) Another form of NLTL. (c) The general soliton waveform on an NLTL.

- Section V: Detailed description of a CMOS prototype that demonstrates a chip-scale operation of the soliton oscillator. This work was briefly reported in [11].

- Section VI: The focus up to Section V is on attaining stable soliton oscillation by controlling unruly solitons in the topology of Fig. 1(b). In this section, we explore the polar opposite, discussing with supporting simulations the case where promotion of the unruly soliton dynamics in the same topology leads to chaotic oscillations.

\section{REVIEW—NLTL AND ELECTRICAL SOLITONS}

\section{A. Solitons}

A pulse traveling in a dispersive medium generally breaks down to multiple sinusoidal waves because different Fourier components propagate at different speeds. Energy initially locally confined in the pulse cannot maintain the spatial localization as the pulse travels.

In contrast, in a nonlinear dispersive medium, the nonlinearity can balance out the dispersion, and a unique pulse exhibiting no dispersion can be propagated. This pulse is known as a soliton [12]-[14]. In the absence of loss, the soliton preserves its exact shape while traveling. When loss is present, the soliton changes its shape as it has to lose energy traveling, but it still maintains spatial localization of energy in the changing pulse shape, not breaking into multiple sinusoids [15]. Other unique properties of solitons will be described shortly in the context of electrical solitons.

Solitons are encountered throughout nature [16]. Water, plasma, mechanical lattices, optical fibers, and magnetic films are examples that can act as nonlinear dispersive media where solitons can be propagated in their respective physical forms.

\section{B. Electrical Solitons}

In electronics, nonlinear transmission lines (NLTLs) serve as nonlinear dispersive media where electrical solitons can propagate in the form of voltage waves [6]. The NLTL is constructed by periodically loading a normal transmission line with varactors (e.g., reverse-biased $p n$ junction diodes or MOS capacitors) [Fig. 2(a)], or alternatively, by arranging inductors and varactors in a 1-D lattice [Fig. 2(b)]. The nonlinearity of the NLTL originates from the varactors whose capacitance changes with applied voltage, while its dispersion arises from its structural periodicity.

Fig. 2(c) shows the general soliton waveform on an NLTL in the absence of loss, which is a periodic train of voltage solitons. This waveform is called the cnoidal wave, and is a solution to what is known as the KdV equation [13]. Many other physical manifestations of solitons, e.g., water wave solitons, plasma solitons, and mechanical lattice solitons are solutions to the KdV equation as well, and the essential properties of electrical solitons are common among these KdV-type solitons. ${ }^{1}$ There are an infinite number of possible cnoidal waves that can form on a given NLTL by interdependently varying amplitude $A$, pulse spacing $\Lambda$, and pulse width $W[A, \Lambda$, and $W$ are with reference to Fig. 2(c)]. Initial or boundary conditions will determine the specific cnoidal wave that can propagate on the NLTL.

Understanding our soliton oscillator, described later, does not warrant detailed mathematical descriptions of the $\mathrm{KdV}$ equation. The following two subsections will rather focus on their physical properties pertinent to this paper.

\section{Propagation and Collision of Electrical Solitons}

In addition to their ability to maintain spatial localizations of energy in pulse shapes, electrical solitons on the NLTL exhibit other unique dynamics [12], [13]. Here we review three relevant dynamical properties.

First, a taller soliton travels faster than a shorter one on the NLTL. Due to this amplitude-dependent speed, if a taller soliton is placed behind a shorter one [Fig. 3(a), top], the taller one will catch up and collide with the shorter one, and move ahead of it after the collision [Fig. 3(a)]. Two other important properties are seen in this collision process. During the collision [Fig. 3(a), middle], the two solitons do not linearly superpose and experience significant amplitude modulations (nonlinear collision). After the collision [Fig. 3(a), bottom], the two solitons recover their original shapes, however, they have acquired a permanent time (phase) shift, shown by the difference between $d_{1}$ and $d_{2}$ in Fig. 3(a): with no time shift, $d_{1}$ and $d_{2}$ would be equal since the time elapse before and after the collision is the same in the figure. The three soliton properties above, i.e., 1) amplitude-dependent speed, 2) amplitude modulation during collision, and 3) phase modulation after collision, are what makes solitons unruly, and cause difficulties in constructing a stable soliton oscillator [Section III].

\section{Forming and Damping of Electrical Solitons}

The previous subsection assumed that electrical solitons were already formed on the NLTL. How do we form solitons on the NLTL in the first place? If a non-soliton wave is launched onto the NLTL, it will change its waveform in the course of propagation to form into a soliton or solitons [13]. More specifically, a non-soliton input close to soliton shape will be sharpened into the soliton while shedding extra energy into a tail ringing [Fig. 3(b), top]. A non-soliton input significantly different from soliton shape will break up into multiple solitons of different amplitudes: the bottom of Fig. 3(b) illustrates an example where a square pulse input on the NLTL breaks up into

\footnotetext{
${ }^{1}$ Light wave solitons belong to a different class of solitons [16].
} 


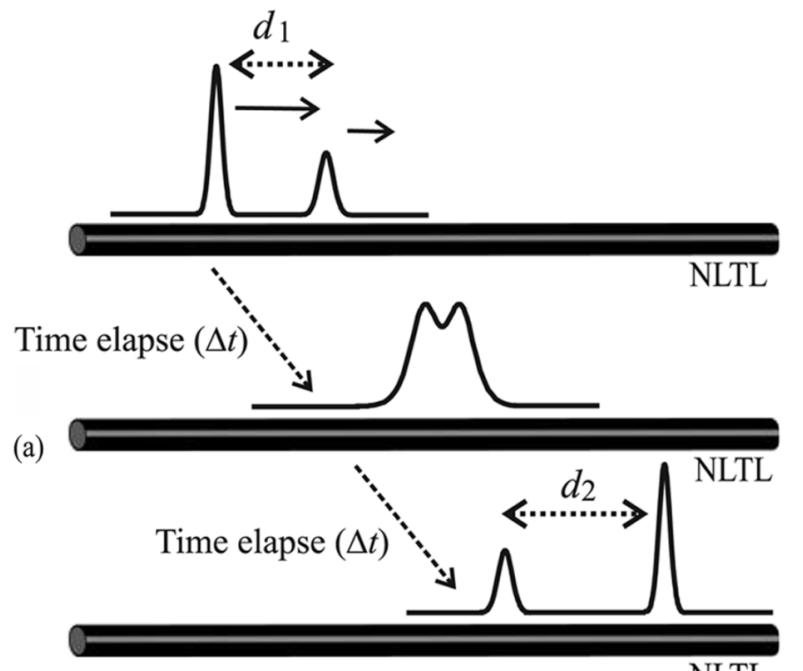

NLTL

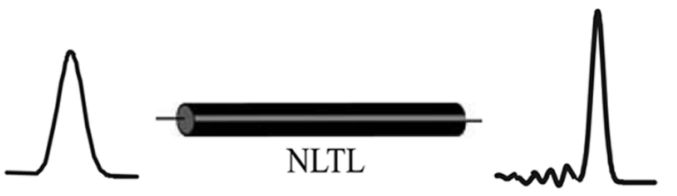

(b)

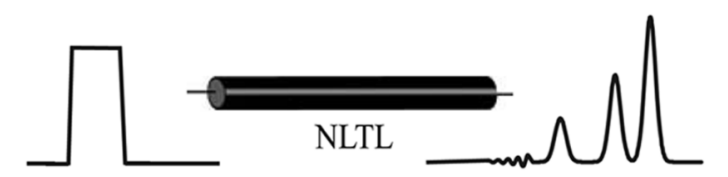

(c)

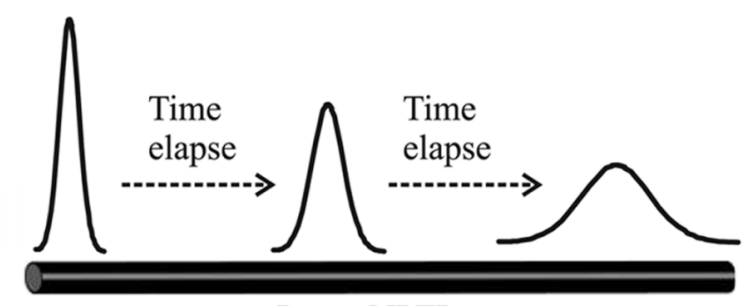

Lossy NLTL

Fig. 3. (a) Amplitude-dependent soliton speed and nonlinear soliton collision on an NLTL. $d_{1} \neq d_{2}$. (b) Transient soliton-forming processes on an NLTL. (c) Soliton damping on a lossy NLTL.

multiple solitons. It is these transient soliton-forming processes [Fig. 3(b), both top and bottom] that have been widely exploited for the past 40 years in the traditional two-port NLTL approach to generate short-duration electrical pulses [6]-[8]. In our oneport soliton oscillator design, the process shown at the top of Fig. 3(b) is beneficial [Section III-D] while the process shown at the bottom of the figure can be detrimental [Section III-B].

Once a soliton is formed through the transient process, it does not undergo any further sharpening or breakup. In the absence of loss, the formed soliton will maintain its exact shape in the course of further propagation. When loss is present, the formed soliton will change its shape as it has to lose energy. The relationships among the amplitude $A$, width $W$, and velocity $v$ of a non-damping soliton (a larger $A$ corresponds to a larger $v$ and a smaller $W$ with specific mathematical relations; the $A-v$ relation is what we previously referred to as amplitude-dependent speed) are preserved even in the damping soliton, and since the damping lowers $A$ in the course of propagation, the damping
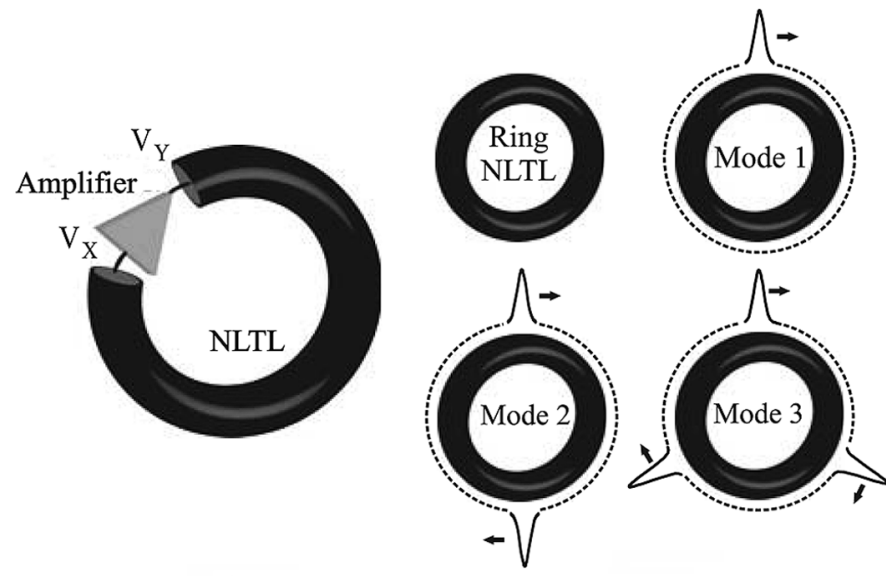

(a)

(b)

Fig. 4. (a) Soliton oscillator topology. (b) Ring NLTL. Mode $1(l=\Lambda)$, Mode $2(l=2 \Lambda)$, Mode $3(l=3 \Lambda)$.

soliton exhibits increasing $W$ and decreasing $v$ [Fig. 3(c)]: this unique soliton damping process is well known as reported by Ott [15]. An important notion is that even a damping soliton maintains spatial localization of energy in its changing pulse shape: the pulse does not break into multiple sinusoids (no dispersion). In addition, damping solitons also retain all the propagation and collision properties described in Section II-C.

As will be seen in Section III-D, the distinctive dynamics between the soliton damping process (pulse widening) and nonsoliton's soliton-forming transient process (pulse sharpening) provide a criterion to determine when a soliton has actually formed on a lossy NLTL.

\section{REVIEW-ELECTRICAL SOLITON OSCILLATOR}

In this section, we review the basic operating principles of the electrical soliton oscillator, which we originally reported in [1]. This is to familiarize this Journal's readership with the still-fresh soliton oscillator concept, and to facilitate the reading of the subsequent sections.

\section{A. Soliton Oscillator Topology}

The soliton oscillator consists of a ring NLTL and a non-inverting amplifier inserted in the ring [Fig. 4(a)]. The ring NLTL supports certain soliton circulation modes determined by the periodic boundary condition, $l=m \Lambda(m=1,2,3, \ldots, l$ : circumference of the ring NLTL; $\Lambda$ : spacing between two adjacent solitons) [Fig. 4(b)]. The amplifier is to enable initial oscillation startup and to compensate loss in steady state (as is commonly done in sinusoidal oscillators [17], [18]). The ultimate goal of this topology is to self-generate and self-sustain one of the soliton circulation modes of Fig. 4(b).

\section{B. Oscillation Instability Mechanisms}

The topology indeed leads to oscillations, self-starting from noise. However, when standard amplifiers are used in the topology, the oscillations tend to be plagued with instability problems, exhibiting significant variations in pulse amplitude and repetition rate [1]. 


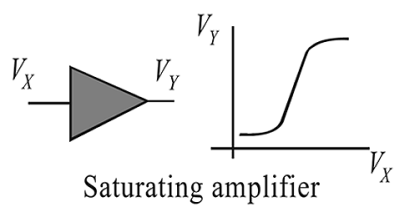

(a)

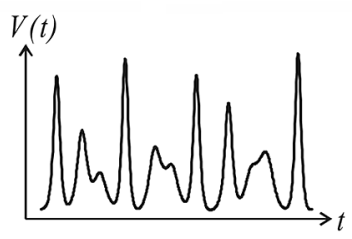

(b)

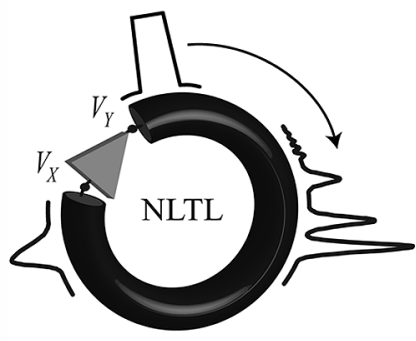

(c)
Fig. 5. (a) A saturating amplifier. (b) Simulated unstable oscillation. (c) Impact of signal saturation.

Generally speaking, the oscillation instability arises because the circular topology not only generates the desired soliton circulation mode, but can also excite parasitic solitons. The desired and parasitic solitons continually collide while co-circulating in the loop due to their generally different amplitudes and resultant speed difference (amplitude-dependent speed, Section II-C). It is these collision events that cause the significant modulations in pulse amplitude and repetition rate (these effects of soliton collisions were explained in Section II-C). To see concretely how colliding solitons are generated, let us examine two cases.

- Case I-Saturating amplifier: Consider the case where a standard saturating, non-inverting amplifier is used in the soliton oscillator [Fig. 5]. The amplifier is biased at a fixed operating point. Assume that a soliton appears at the amplifier input at a certain time $\left[V_{X}\right.$, Fig. $\left.5(\mathrm{c})\right]$. This soliton, after passing through the amplifier, will turn into a square pulse due to the saturation by the amplifier [ $V_{Y}$, Fig. 5(c)]. As seen in Section II-D, the square pulse will break up into several solitons of different amplitudes/speeds while traveling down the NLTL, eventually reappearing at the amplifier input at different times. This process repeats itself, creating many solitons with various amplitudes in the loop. These solitons circulate with different speeds (amplitude-dependant speed) and hence, continually collide with one another, causing time shifts and amplitude variations. The result is unstable oscillation [Fig. 5(b)]. The signal saturation is a clear cause of the soliton collision events and the resultant oscillation instability.

- Case II-Linear amplifier: The discussion above suggests that one might be able to attain a stable soliton oscillation if signal saturation is mitigated by using a linear amplifier [Fig. 6(a)] in the circular topology of Fig. 4(a). Ballantyne et al. [19], [20] indeed implemented such a system, where a periodic train of solitons was seen. With minor $(\sim 2 \%)$ changes to loop parameters such as gain, however, multiple pulses appeared in the oscillator and collided with one another, causing once again oscillation instabilities [Fig. 6(b)]. This shows the lack of robustness, reproducibility, and controllability in the soliton oscillator using the linear amplifier.

This second case suggests that the saturation reduction is a necessary but not a sufficient condition to stabilize the oscilla-

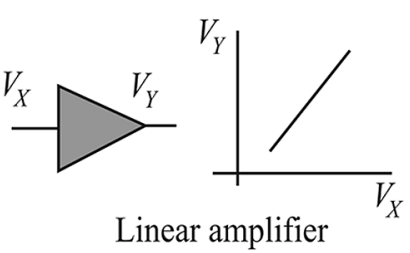

(a)

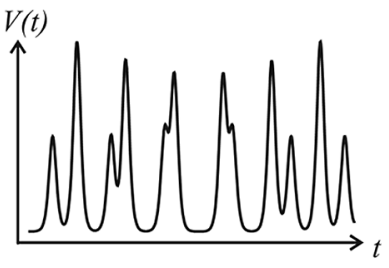

(b)
Fig. 6. (a) A linear amplifier. (b) Depiction of oscillations with pulse amplitude and repetition rate variations [19], [20].

tion and ensure reproducibility, and there are other mechanisms that cause soliton collision events. Two other mechanisms we identified in [1] are perturbations and multi-mode oscillations. First, any small ambient perturbation (e.g., noise) can grow into parasitic solitons in the soliton oscillator due to gain provided by the amplifier. The desired soliton circulation mode and parasitic solitons will propagate at different speeds due to their generally different amplitudes, colliding and building up oscillation instabilities. Second, various soliton circulation modes of Fig. 4(b) with generally different amplitudes can co-circulate in the loop at different speeds, leading to soliton collision events again and, hence, unstable oscillation.

Summarizing, the soliton oscillator of Fig. 4(a) readily lends itself to production of multiple colliding solitons. Three main causes are:

- signal saturation;

- ambient perturbations;

- multi-mode oscillation.

The key to our success in stable soliton oscillator design in [1] was to identify these three instability mechanisms and to develop an amplifier for the soliton oscillator of Fig. 4(a), which not only provides gain but also incorporates functionalities to suppress the three instability mechanisms.

\section{Taming Electrical Solitons with an Amplifier}

In [1], we attained the stabilizing amplifier by incorporating an adaptive bias control in a standard saturating amplifier. In this subsection, we will explain how the adaptive bias control can be used to simultaneously suppress the three instability mechanisms.

Fig. 7(a) shows the transfer curve of the saturating amplifier, which can be divided into the attenuation, gain, and saturation regions based on the curve's tangential slopes. At the initial startup, the amplifier is biased at $\mathbf{A}$ in the gain region so that ambient noise can be amplified to initiate oscillation startup. As the oscillation grows and forms into a soliton train, the DC component of the oscillation signal increases [Fig. 7(b)]. This increase in the DC component is used to adaptively lower the amplifier bias [dashed arrow, Fig. 7(c)]. The reduced bias corresponds to an overall gain reduction, since a portion of the pulse enters the attenuation region. The bias point continues to move down on the curve until the overall gain becomes equal to the system loss, finally settling at steady-state bias $\mathbf{B}$.

In steady state [Fig. 7(c)] with bias $\mathbf{B}$ situated in the attenuation region, the three instability mechanisms are prevented simultaneously. First, the reduced bias ensures that the peak 


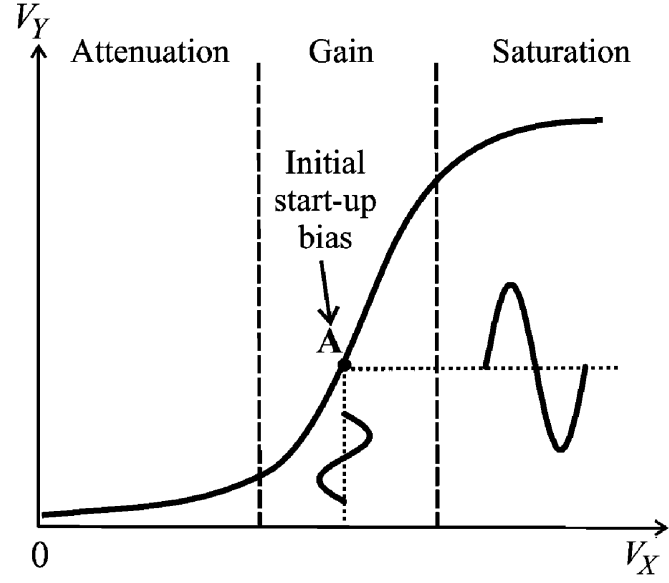

(a)

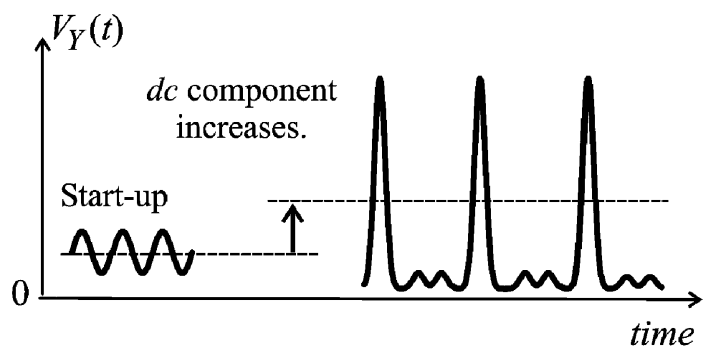

(b)

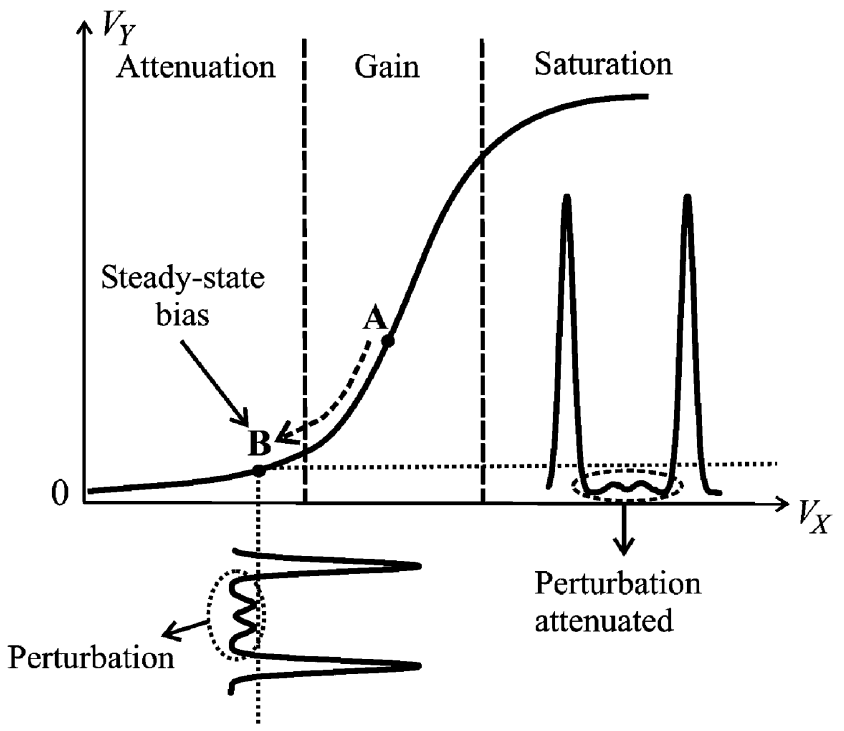

(c)

Fig. 7. (a) Transfer curve of our stabilizing amplifier. $\mathbf{A}$ is initial start-up bias. (b) DC component of $V_{Y}$ increases as the oscillation grows into a soliton train. (c) Increased DC component is used to lower the bias of the amplifier, leading to steady-state bias $\mathbf{B}$.

portions of input pulses do not enter the saturation region, mitigating signal saturation. Second, with the reduced bias in steady state [Fig. 7(c)], the input soliton train is placed across the attenuation and gain regions, causing small ambient perturbations such as noise and tail ringings around the bias to be attenuated. Note that the perturbation rejection is accomplished while maintaining gain for the main portions of input pulses to compensate loss. This threshold-dependent gain-attenuation mechanism is widely employed in mode-locked lasers in optics,

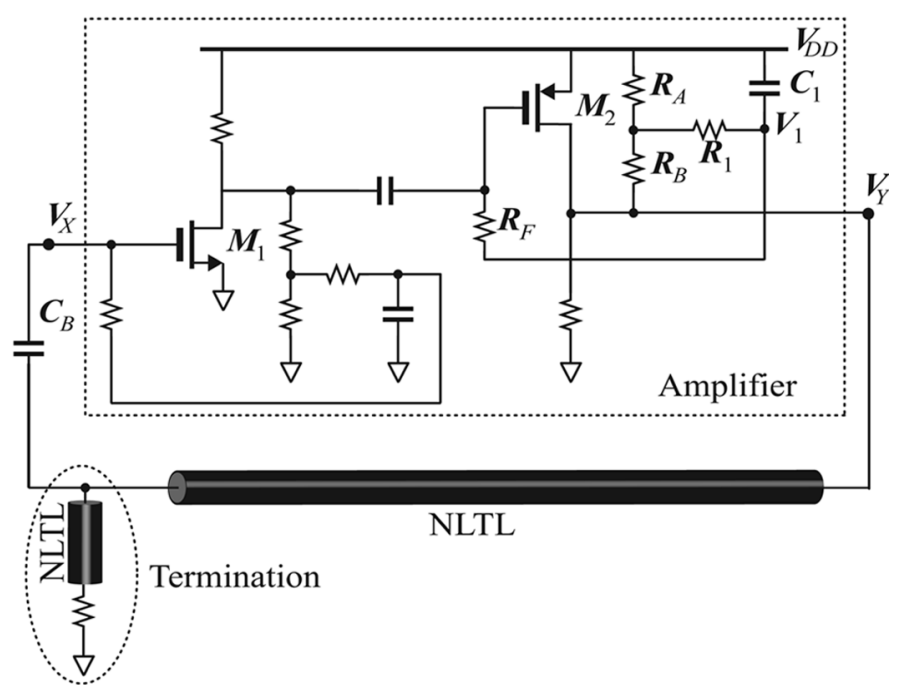

Fig. 8. Circuit schematic of the first soliton oscillator prototype.

where it is known as saturable absorption [21], but was originally introduced in electronics domain by Cutler for his linear pulse oscillator [22], [1]. Note that the nonlinearity in the lower portion of the transfer curve is exploited to suppress the second instability mechanism (perturbations) while the nonlinearity in the upper portion is avoided to mitigate the first instability mechanism (signal saturation).

The third instability mechanism (multi-mode oscillation) is also suppressed via the adaptive bias control. In steady state, a higher mode [among various soliton modes of Fig. 4(b)] has a larger DC component and therefore its corresponding steady-state bias sits farther down on the transfer curve, making the mode receive less overall gain. This mode-dependent gain allows for single-mode selection [1].

\section{First Discrete Prototype}

To demonstrate the concept of the stable electrical soliton oscillator, we constructed the first prototype at the discrete level [1]. The circuit schematic is shown in Fig. 8. The amplifier shown within the dashed box incorporates the adaptive bias control to perform the functionality described with Fig. 7. The amplifier consists of two functionally equivalent, complementary inverting stages, one built around an nMOS transistor, $M_{1}$, and the other around a pMOS transistor, $M_{2}$, which, when taken together, form a non-inverting amplifier. The pMOS stage works as follows. The amplifier output, $V_{Y}(t)$, is sensed by the $R_{A}-R_{B}$ voltage divider, and then is integrated by the $R_{1}-C_{1}$ low pass filter. The integrated voltage, $V_{1}$, represents a scaled DC component of $V_{Y}(t)$. This DC component is fed to the gate of $M_{2}$ through resistor $R_{F}$ to set its bias. As the DC level of $V_{Y}$ increases, $V_{1}$ will rise, reducing the gate-source voltage of $M_{2}$, effectively lowering its bias. A similar argument applies to the nMOS stage. Combining the two stages, the effective bias of the amplifier at the input is reduced as the DC level of $V_{Y}$ increases, performing the adaptive bias control to achieve the functionality of Fig. 7.

The NLTL of Fig. 8 has a termination at the amplifier input to minimize reflection back to the NLTL, which could otherwise significantly perturb the soliton oscillation. Perfect termination of an NLTL is challenging since its characteristic impedance 
(a)

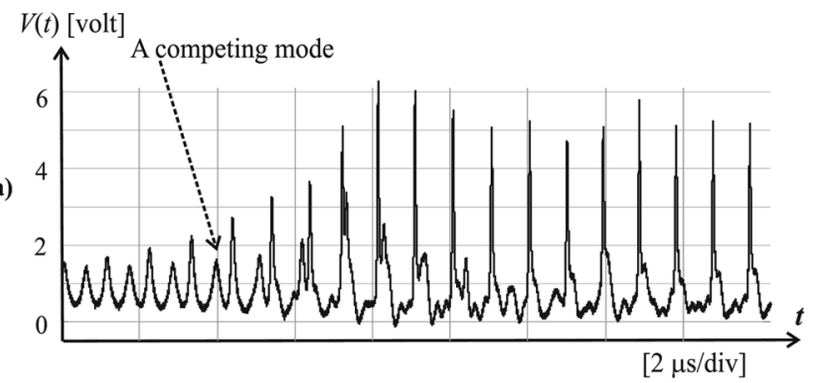

(b)

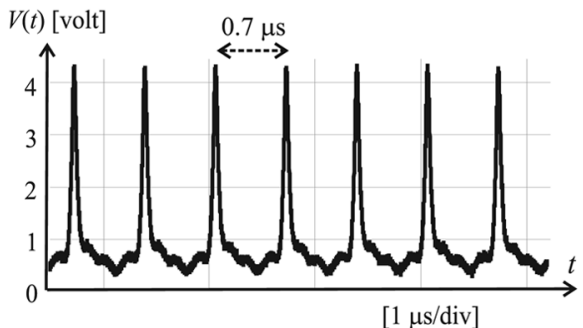

(c)

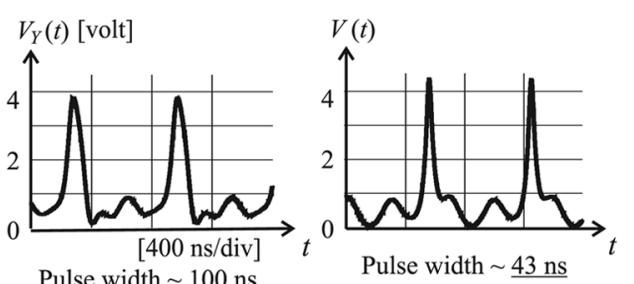

$$
\text { Pulse width } \sim \underline{100 \mathrm{~ns}}
$$

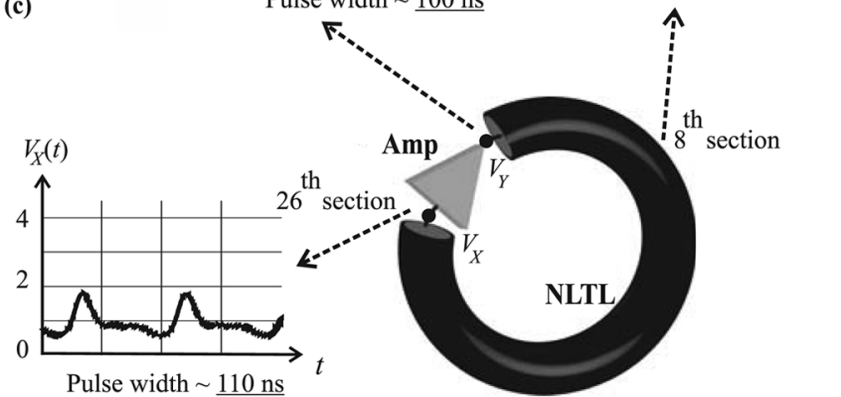

Fig. 9. (a) Measured startup transient [1]. (b) Steady-state soliton oscillation [1]. (c) Measured steady-state oscillation at various points [1]. FWHM pulse widths are measured.

changes with voltage. Our termination consists of an intentionally high-loss section of NLTL and a resistor whose value is the average of the characteristic impedance seen by a desired signal. Due to the imperfect termination, reflections do occur, but they are small enough to be attenuated by the perturbation rejection mechanism incorporated in the amplifier.

Fig. 9(a) shows measured oscillation startup at the 8th section of the NLTL (total $22 L C$ sections). The oscillator starts by amplifying ambient noise creating a small oscillation initially, eventually growing into a steady-state soliton train. During this process, another competing mode is clearly seen. It first grows with time, but is eventually suppressed by the stabilizing mechanism of the amplifier. In the figure, one can also observe that the shorter pulse (competing mode) propagates at a different speed than the taller pulse (surviving mode): in this time-domain measurement at the fixed point on the NLTL, the shorter pulse originally behind the taller pulse catches up with the taller pulse and eventually moves ahead of it after collision. In the space domain, this corresponds to the taller pulse propagating faster than the shorter pulse, a key signature of solitons [Section II-C].

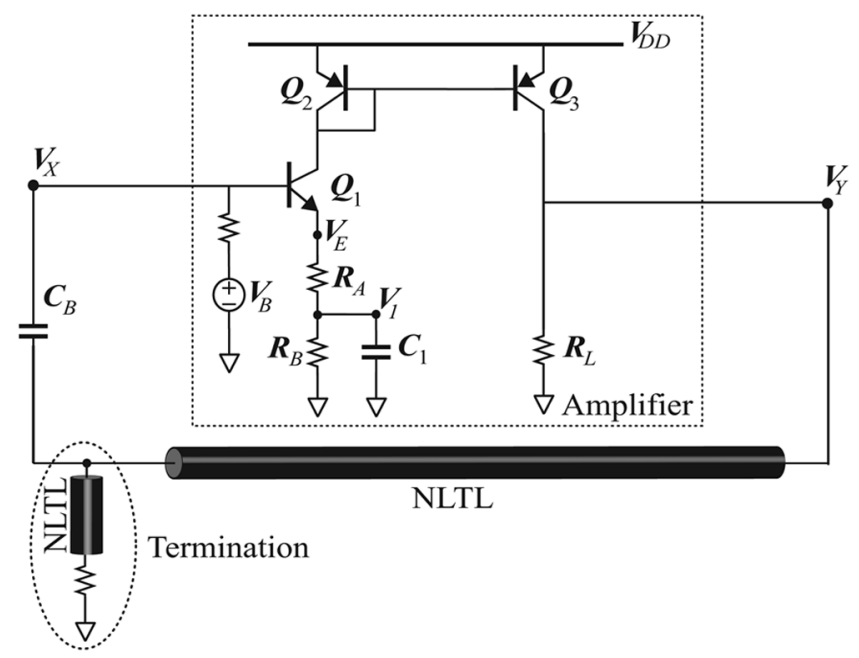

Fig. 10. Circuit schematic of the second soliton oscillator prototype.

Fig. 9(b) shows a steady-state soliton train measured at the 8th section on the NLTL (total $30 L C$ sections). The amplitude and pulse repetition rate remained stable. When perturbed with large external signals, the oscillation always returned to the same steady-state soliton train. Additionally, for a given set of circuit parameters, every startup led to the same steady-state oscillation. These demonstrate the same level of robustness and stability found in standard sinusoidal oscillators [17], [18].

One of the most fascinating dynamics of the soliton oscillator can be observed by following the pulse around the oscillator loop in steady state. Fig. 9(c) shows such spatial dynamics measured at three different positions on the NLTL. At the amplifier output, the pulse is not an exact soliton, and, hence, sharpens into a soliton traveling down the NLTL. Once the soliton is formed at the 8th section, it does not further sharpen since it is now a soliton. Instead, the soliton damping [Section II-D] becomes the dominant process as the soliton further travels down the NLTL, lowering in amplitude and velocity while increasing in width. It is this clear existence of the transition point (8th section) between the pulse sharpening and widening (damping) that shows the formation of the soliton at that transition point.

\section{E. Second Discrete Prototype}

The soliton oscillator is a general concept and can be scaled in frequency. We built the second discrete prototype at a higher frequency range. The preliminary result was presented in [1]. Here, we provide its full description.

The oscillator schematic is shown in Fig. 10. The bipolar junction transistor (BJT) amplifier inside the dashed box consists of a common emitter amplifier with emitter degeneration and a signal inversion stage. While the MOS amplifier of Fig. 8 adjusted its bias based on its output signal, this BJT amplifier adjusts its bias based on its input signal. Specifically, it produces its input signal's scaled DC component at $V_{1}$ via the $R_{A}-R_{B}$ voltage divider and the $R_{B}-C_{1}$ low-pass filter. Also differently from the MOS amplifier, the BJT amplifier's input bias (base of $Q_{1}$ ) is fixed. In the initial startup transient, the fixed bias of the amplifier is in the gain region [top, Fig. 11]. As 


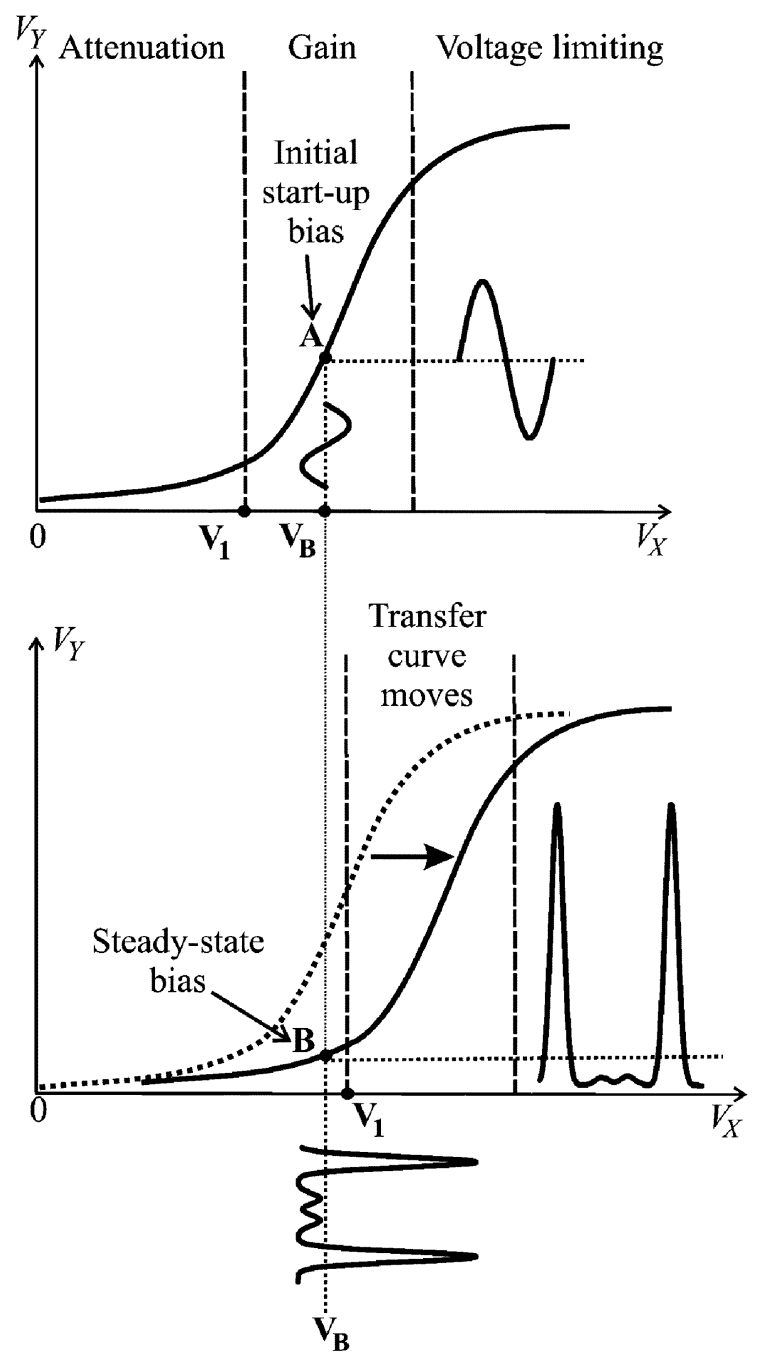

Fig. 11. Effective adaptive bias control in Fig. 10.

the oscillation grows and forms into a pulse train, $V_{1}$ increases, which moves the amplifier transfer curve towards right [bottom, Fig. 11], placing the fixed bias in the attenuation region. As a result, in steady state, the input pulse is placed across the gain-attenuation regions. Summarizing, while this amplifier moves the transfer curve instead of the amplifier bias, in steady state, it places the pulse train just like in Fig. 7(c), hence achieving all of the three stability mechanisms. The measured steady-state soliton oscillation from the second prototype is shown in Fig. 12. The periodic soliton train clearly seen remained as stable as the first prototype.

With the soliton oscillator concept review complete in this section, we will now move on to new contributions.

\section{FURTHER EXPERIMENTAL StUdies ON THE SOliton OSCILLATOR DYNAMICS}

\section{A. Existence of a Limit Cycle}

In [1], the stability of the soliton oscillator was demonstrated by steady-state oscillations that were robust to ambient and external perturbations. The key character of any stable oscillator is the existence of a limit cycle in phase space. For instance, for an $L C$ oscillator whose voltage across the $L C \operatorname{tank}$ is $V$, the

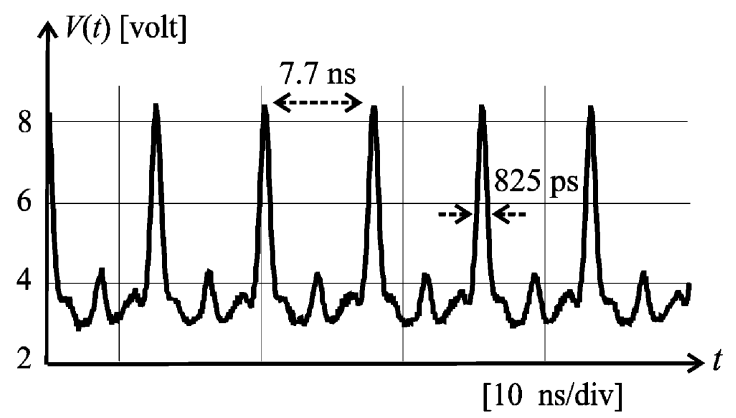

Fig. 12. Soliton oscillation from the second prototype [1].

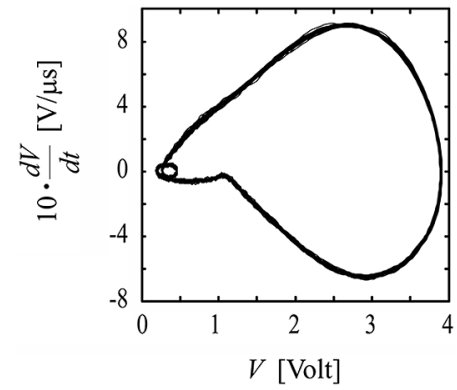

(a)

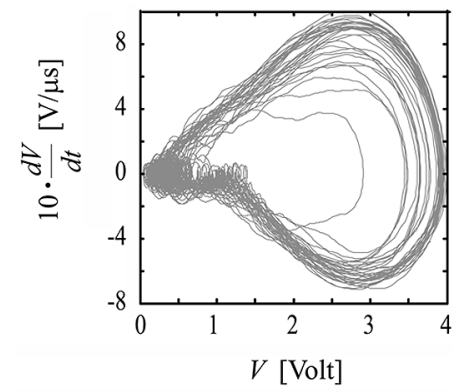

(b)

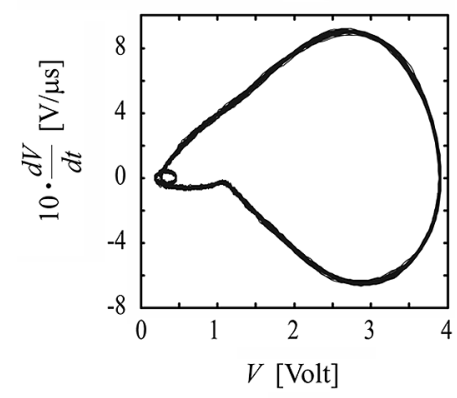

(c)

Fig. 13. (a) Steady-state trajectory of $(V, d V / d t)$. (b) Transient response to a significant disturbance. (c) Steady-state trajectory after the effect of perturbation has settled.

steady-state oscillation maps onto an ellipse-like closed trajectory (limit cycle) in a 2-D $(V, d V / d t)$ space (phase space). Regardless of the initial condition or after perturbations, the oscillation point $(V, d V / d t)$ always ends up on the limit cycle in steady state.

Here, we experimentally demonstrate the existence of a limit cycle in the soliton oscillator, using the first discrete prototype [Section III-D]. The low-frequency discrete prototype is used to facilitate a large perturbation of the system. Fig. 13(a) shows the trajectory of the steady-state oscillation point $(V, d V / d t)$ in the 


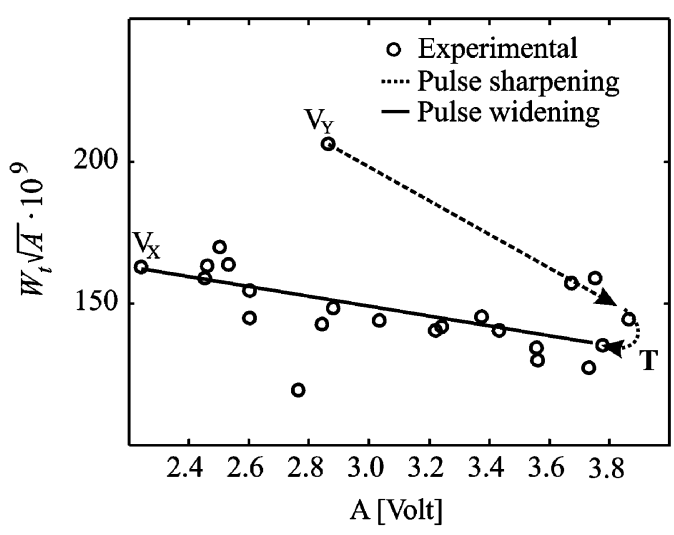

Fig. 14. $W_{t} \sqrt{A}$ versus $A$.

2-D phase space where $V$ is the voltage taken from the 7th node on the NLTL. ${ }^{2}$ After a significant perturbation of the soliton oscillator using an external pulsed source, the oscillation trajectory in the phase space deviates from the steady-state trajectory of Fig. 13(a) for many cycles as depicted in Fig. 13(b), but it eventually settles back to the same original steady-state trajectory [Fig. 13(c)]. This experiment demonstrates that the steady-state trajectory of Fig. 13(a) is the limit cycle of the soliton oscillator.

\section{B. Spatial Dynamics Revisited}

The spatial dynamics of the first prototype shown in Fig. 9(c) exhibited a clear transition from pulse sharpening to pulse widening. There we reasoned that the sharpening and widening correspond to soliton-forming and soliton-damping processes, respectively.

To confirm this reasoning, the temporal pulse width $W_{t}$ and pulse amplitude $A$, measured at each section of the NLTL of the first prototype in its steady state, are plotted in the $W_{t} \sqrt{A}$ versus $A$ plane. See Fig. 14. ${ }^{3}$ This specific choice of $x-y$ axes is just one way of showing how the pulsewidth and amplitude change as the pulse circles around the oscillator loop. Fig. 14 shows that the initial pulse sharpening process and the following pulse widening process trace two distinct trajectories, $V_{Y}$ to $\mathbf{T}$ (dashed line) and $\mathbf{T}$ to $V_{X}$ (solid line), respectively. This clearly suggests that the pulse in the sharpening process and that in the widening process are of fundamentally different nature.

$A$ and $W_{t}$ of a soliton on the NLTL are related by [14]

$$
W_{t} \sqrt{A}=-\frac{2 \sqrt{b} \sqrt{L C_{0}}}{\sqrt{6}} \cdot A+\frac{3}{\sqrt{6 b}} \cdot \sqrt{L C_{0}}
$$

where $L$ is the single inductance in the NLTL, and $b$ and $C_{0}$ are derived from the varactor model $C(v) \approx C_{0} \cdot(1-2 b v)$ where $v$ is the applied voltage. As mentioned in Section II-D, even in a damping soliton, the $A-W_{t}$ relation above is preserved [15], making a plot of $W_{t} \sqrt{A}$ versus $A$ a straight line. Now as can be seen from Fig. 14, the data points during the pulse widening can be placed roughly on the solid straight line. Extracted $b, C_{0}$, and

\footnotetext{
${ }^{2}$ The real phase space is $2 N$-dimensional where $N$ is the number of $L C$ sections in the NLTL. The dynamics in the 2-D "pseudo"-phase-space, however, reasonably well reflects the entire system dynamics.

${ }^{3}$ The set of circuit parameters used in this specific experiment is different from those used for the experiment to obtain Fig. 9(c).
}

$L$ from this solid line deviate from the real values by $20 \%$, which arises from the inaccurate first-order modeling of the varactor, but the general trend clearly shows that the pulse during the widening is indeed a soliton that is damping. In contrast, the data during the sharpening process are far off from the solid straight line, showing that the pulse during the sharpening is not a soliton (it is sharpening into a soliton).

\section{CMOS ELECTRICAL SOLITON OSCILLATOR}

To demonstrate chip-scale operation of the soliton oscillator, we implemented the circuit of Fig. 8 in $0.18-\mu \mathrm{m} \mathrm{CMOS} \mathrm{process.}$ Here, we detail its design, construction, and measurements (this work was briefly reported in [11]).

\section{A. Design Procedure}

In the absence of loss, the temporal soliton width $W_{t}$ and the soliton repetition rate $f$ of the $n$th soliton circulation mode on the ring NLTL [Fig. 4(b)], consisting of N LC sections, are given by [16]

$$
\begin{aligned}
W_{t} & =\frac{3-2 b A}{\sqrt{6 b A}} \cdot \sqrt{L C_{0}} \\
f & =\frac{1}{\sqrt{L C_{0}}} \cdot \sqrt{\frac{3}{3-2 b A}} \cdot \frac{n}{N}
\end{aligned}
$$

where $A$ is the soliton amplitude. These equations are very useful as initial design guidelines, but one should eventually resort to circuit simulations for the following reasons. First, $W_{t}$ and $f$ in (2) and (3) are functions of not only the component values, but also the soliton amplitude, $A$, which is difficult to express in terms of component values. Second, the delay in the amplifier will result in the pulse repetition rate $f$ smaller than what is predicted by (3). Third, the equations above assume that the soliton waveform is the same everywhere on the NLTL in steady-state oscillation, but this is untrue as we saw in Fig. 9(c).

The simulated target pulse repetition rate and pulsewidth of the chip-scale soliton oscillator were around $1 \mathrm{GHz}$ and a few hundred picoseconds, respectively. These relatively lower speeds were chosen to facilitate time-domain measurement of this first chip-scale proof-of-concept using a real-time oscilloscope.

\section{B. Construction of the Chip-Scale Soliton Oscillator}

Fig. 15 shows a micrograph of the implemented chip-scale soliton oscillator, which consists of an IC mounted on an in-house fabricated glass substrate. The IC contains the stabilizing amplifier and the varactors ( $p n$-junction diodes) of the NLTL. The NLTL inductors were created by bonding gold wires back and forth between the pads on the IC and metallic pads on the glass substrate. Bonding was done by using an automated bonding machine to ensure consistency in wire length. The estimated inductance variation from inductor to inductor was less than 5\%. Magnetic couplings between adjacent wires estimated via electromagnetic simulations were taken into account in choosing proper lengths of the bonding wires to generate a desired inductance. The bonding wires were used as NLTL inductors to test various different NLTL characteristics (inductance value and NLTL length) in the soliton oscillator via 

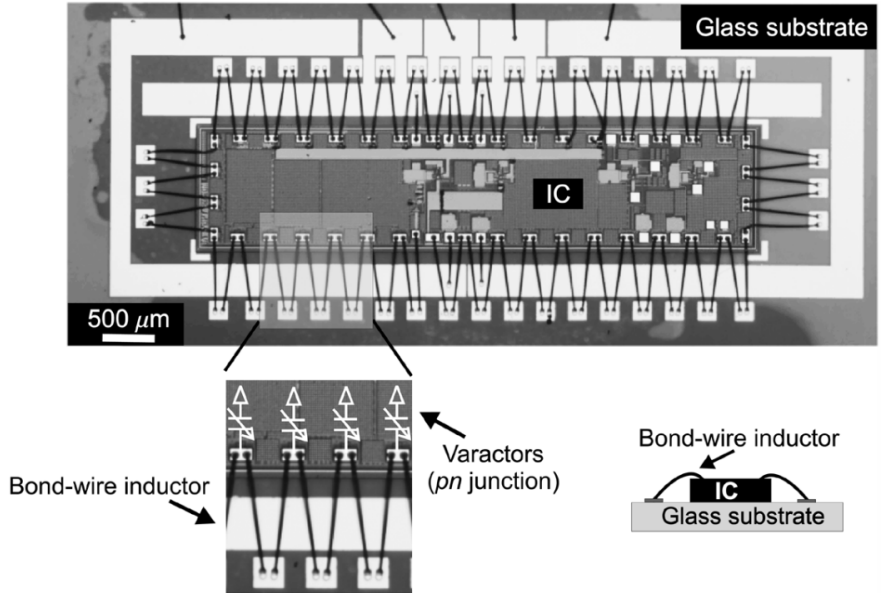

Fig. 15. Micrograph of the chip-scale soliton oscillator, consisting of a CMOS IC mounted on a glass substrate.

post-fabrication adjustment in this initial stage of the chip-scale prototype development. However, the bonding pads on the IC introduce parasitic linear capacitors, which compromise the nonlinearity of the NLTL.

\section{Measurement Results}

The chip-scale system of Fig. 15 self-starts from noise, leading to self-sustained stable soliton oscillation. Fig. 16(a) shows the soliton train produced, measured at one point on the NLTL using an Agilent 54855A real-time oscilloscope. The soliton train has a pulse repetition rate of $1.14 \mathrm{GHz}$ and a pulsewidth (FWHM) of 293 ps. Several different samples of the chip-scale prototype were obtained by varying the NLTL characteristics via bond wire adjustment. These samples had a pulse repetition rate ranging from 0.9 to $1.9 \mathrm{GHz}$ and pulse width ranging from 293 to 400 ps.

Fig. 16(b) shows the steady-state voltage signals measured at three different nodes on the NLTL. This is to show how the pulse shape changes as it circulates in the chip-scale oscillator loop in steady state, as we did the same with the first prototype [Fig. 9(c)]. The spatial dynamics seen in Fig. 9(c) in the discrete prototype, i.e., soliton forming first, followed by soliton damping, is again observed here, suggesting the universality of the spatial dynamics.

Note that in this specific chip-scale implementation, the degree of the pulse compression in the soliton forming process (429 ps $\rightarrow 385$ ps: width reduction by a factor of $89 \%$ ) is not as significant as in the case of the first discrete prototype [Fig. 9(c): $100 \mathrm{~ns} \rightarrow 43$ ns: width reduction by a factor of $43 \%]$. This is because the nonlinearity of the NLTL is compromised by the parasitic linear capacitance of the bonding pads, which were necessary in this specific implementation using bonding wires to construct the NLTL. In future implementations, the inductive elements can be obtained using on-chip planar transmission lines [18] to reduce the stray elements and to maximize NLTL's nonlinearity.

\section{Discussions and Future Directions}

The minimum pulsewidth of 293 ps achieved in our latest chip-scale soliton oscillator prototype is not a record number (a)

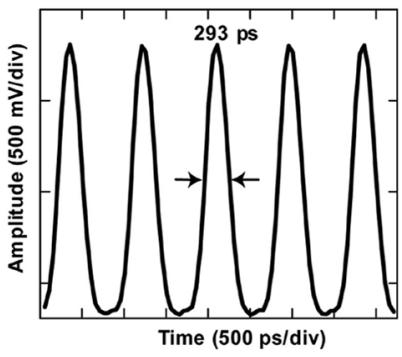

(b)

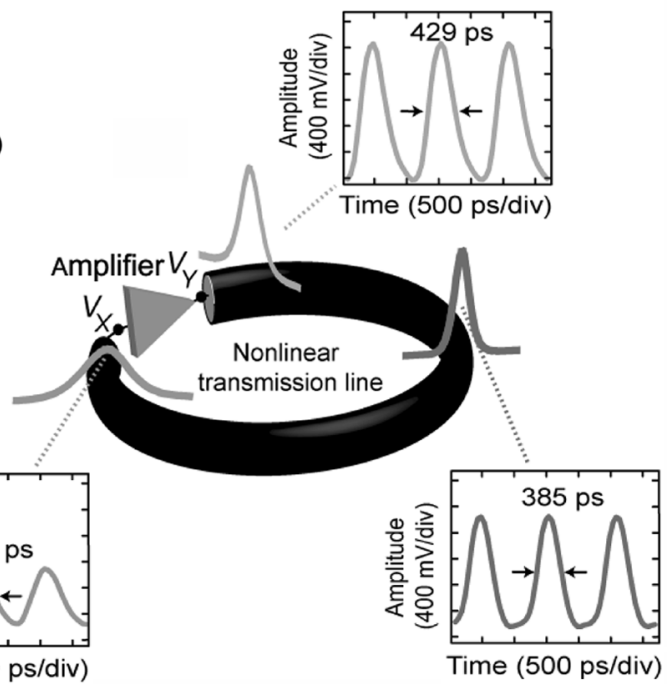

Fig. 16. (a) Measured steady-state soliton oscillation of the chip-scale soliton oscillator. (b) Measured spatial dynamics of the chip-scale soliton oscillator in steady state.

as compared to the state-of-the-art pulse generation circuits, including the ultrafast two-port NLTL [8]. The purpose and value of our work so far lie in the clear demonstration of the stable electrical soliton oscillator concept and its feasibility for chip-scale operation. We intentionally chose rather slower operation speeds to facilitate the in-depth study of this new class of circuits using the real-time oscilloscope. Now with both the concept and chip-scale operation firmly demonstrated, the soliton oscillator, especially its NLTL, can be quickly scaled to a smaller size to significantly enhance the speed. For instance, the ultrafast two-port GaAs NLTLs in [8] (480-fs pulse rise time) can be used in a GaAs soliton oscillator IC to significantly reduce the soliton width. (Although the first chip-scale prototype was in CMOS, this was due to the availability of the technology at the time of implementation, and the chip-scale system is extendable to other semiconductor technologies.)

Using such an ultrafast NLTL in the soliton oscillator raises an important question on the impact of the amplifier bandwidth on the minimum soliton width. While the propagation of a 1-ps wide pulse on the standalone NLTL is feasible [8], amplifiers, even in the state-of-the-art solid-state technologies, cannot provide bandwidth for such a sharp pulse. The experimental results shown with Figs. 9(c) and 16(b) clearly suggest, however, that the soliton forming process (pulse sharpening) on the NLTL after the output of the amplifier may be able to overcome the bandwidth limitation of the amplifier, and hence, it may be feasible to achieve a 1-ps pulsewidth using the NLTL of [8] despite 


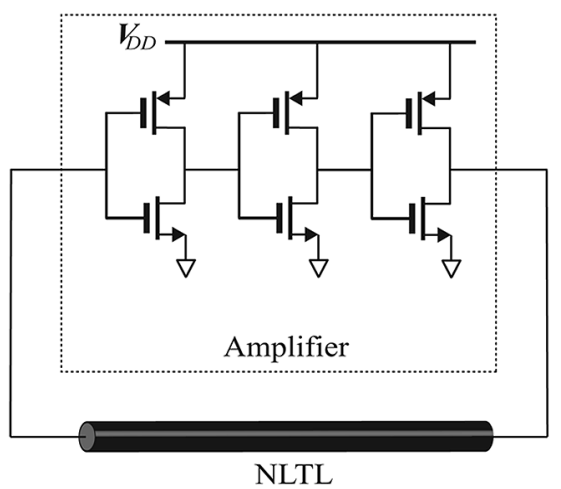

Fig. 17. Chaotic soliton oscillator.

the relatively slower amplifier. The explicit demonstration of this interesting possibility remains an open question, and would be a natural extension of this work.

Final remark: the soliton oscillator of Fig. 4(a) used so far to prove stable soliton oscillation uses a lumped amplifier. The design of a distributed soliton oscillator where gain is provided all along the NLTL could be an important direction in the further development of the oscillator.

\section{CHAOTIC SOLITON OSCILLATOR}

We have so far focused on stable soliton oscillation by developing the amplifier that can control the unruly soliton behaviors. Now let us look at the opposite end of the spectrum, and consider if one can attain an extreme oscillation instability, or chaos, by promoting the unruly soliton dynamics. The discussion in Section III-B suggests that one way of promoting the soliton's unruly behavior is to increase the signal saturation in the amplifier in the soliton oscillator of Fig. 4(a). This could intensify oscillation instabilities (variations in pulse amplitude and repetition rate) through increased soliton collision events, possibly leading to chaotic oscillations, as the authors suggested in [23].

To examine the possibility of chaotic oscillation, we consider the circuit of Fig. 17. This is of the same topology as the original soliton oscillator of Fig. 4(a) in that an amplifier and an NLTL are combined in a ring. But since neither preservation of pulse polarity nor stability mechanism is required in the amplifier to attain chaos, we opted for an inverting amplifier consisting of three CMOS inverter stages for simplicity. The inverting amplifier also facilitates signal saturation and obviates the need for a DC-blocking capacitor like $C_{B}$ in Figs. 8 and 10.

Using Cadence, we performed transistor-level transient simulations for the circuit (24 $L C$ sections for the NLTL; $L=220 \mathrm{nH}$ with a $1.3 \Omega$ series resistance. The complex varactor model used in the simulation, if Taylor-expanded, gives $C(v) \sim C_{0}(1-$ $2 b v$ ) with $C_{0}=30 \mathrm{pF}$ and $b=0.56$ ). Fig. 18(a) shows simulated voltage $V(t)$ at one node on the NLTL, while Fig. 18(b) shows its Fourier transform. The unstable oscillation in time domain and the broad spectral distribution hint at chaotic oscillation. Figs. 18(c) and (d) show the oscillation trajectories in the $(V, d V / d t)$ phase space for two different time spans: as the time span increases [Fig. 18(d)], the trajectory fills more of what appears to be a bounded region. This phase space dynamics once again indicates that the oscillation may be chaotic. (a)

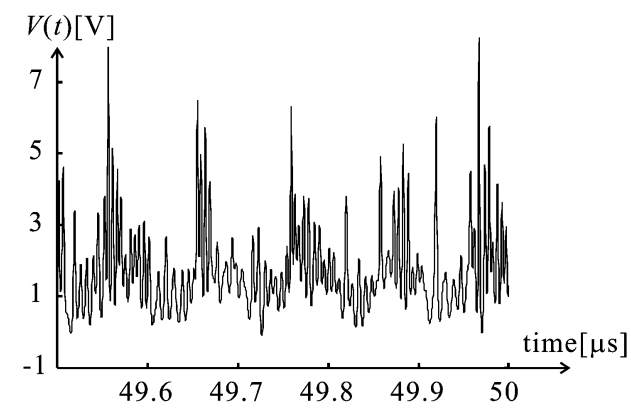

(b)

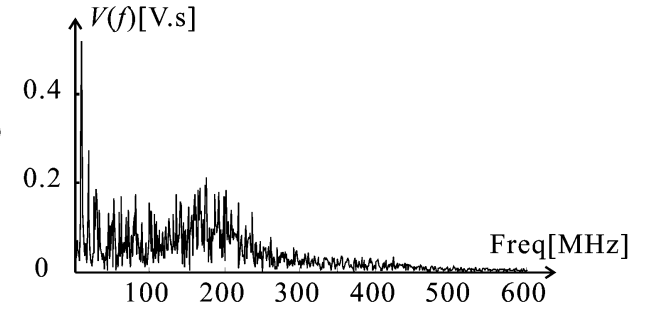

(c)

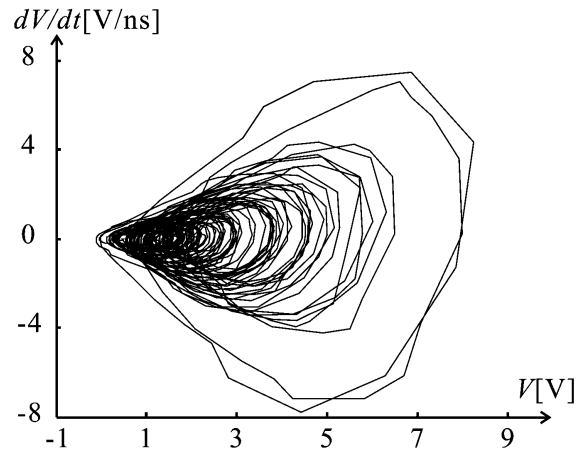

(d)

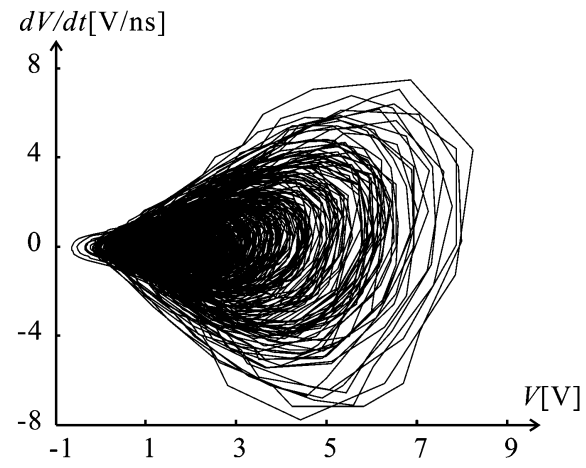

Fig. 18. Simulation of the circuit of Fig. 17. (a) Time-domain signal, $V(t)$, at one node on the NLTL. (b) Fourier transform of $V(t)$. (c) Phase space dynamics from $t=49.5 \mu \mathrm{s}$ to $t=50 \mu \mathrm{s}$. (d) Phase space dynamics from $t=47.5 \mu \mathrm{s}$ to $t=50 \mu \mathrm{s}$.

The hallmark property of a chaotic system is that the distance $d(t)$ of two phase-space points initially proximate to each other (very close initial condition: $d(0) \rightarrow 0$ ) will grow exponentially with time. We simulated a model mimicking the circuit of Fig. 17 in Matlab and Fig. 19 shows the evolution of $\log (d(t) / d(0))$ where $d(0)=10^{-10}$. It increases linearly with time (positive slope), confirming that $d(t)$ exponentially grows and the oscillator is indeed chaotic. (The slope is the Lyapunov exponent, whose positiveness is a sufficient indicator of chaos in general [24].)

Electrical chaos generators are of significant engineering interest due to their potential use in applications such as encrypted chaotic communications [25]. While chaos generation circuits 


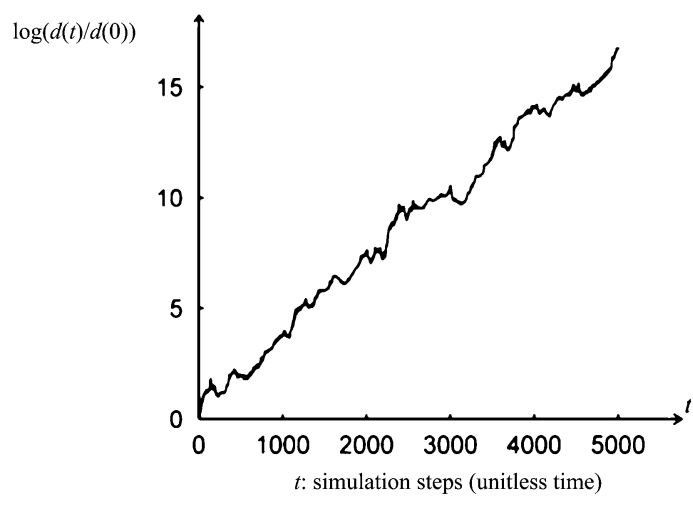

Fig. 19. Simulated $\log (d(t) / d(0))$ versus $t$.

abound, the chaotic soliton oscillator may have an edge with its potentially very large bandwidth owed to the NLTL's soliton compression capability [6]-[8].

\section{CONCLUSION}

While the hegemony of sinusoidal signals for high-frequency signal processing in electronics will undoubtedly last into the foreseeable future, certain non-sinusoidal signals, such as shortduration pulses and chaotic signals, may further enrich the scope and capacity of modern electronics. The electrical soliton oscillator presented in this paper can self-generate both a stable train of short soliton pulses and a chaotic signal, by taming or encouraging the unruly nature of solitons. The soliton's superb ability of pulsewidth compression and resultant large bandwidth may give the edge to the electrical soliton oscillator over other pulse and chaos generation circuits. This prospect is brightened by the notion that nature's most intricate and brilliant circuit, the human brain, utilizes soliton-like neuron impulses, and often, their chaotic behaviors.

\section{ACKNOWLEDGMENT}

The authors thank M. DePetro, W. Andress, A. Belabbas, and Prof. R. Brockett of Harvard University for discussions, and L. DeVito and R. Sullivan of Analog Devices for help with the bonding-wire NLTL construction. Prof. T. H. Lee of Stanford University shared invaluable insights for the chaotic soliton oscillator work. The authors also thank Prof. C. Sodini and K. Ryu of MIT for their help with varactor characterizations.

\section{REFERENCES}

[1] D. S. Ricketts, X. Li, and D. Ham, "Electrical soliton oscillator," IEEE Trans. Microw. Theory Tech., vol. 54, no. 1, pp. 373-382, Jan. 2006.

[2] M. Kahrs, "50 years of RF and microwave sampling," IEEE Trans. Microw. Theory Tech., vol. 51, no. 6, pp. 1787-1805, Jun. 2003.

[3] R. Y. Yu, M. Reddy, J. Pusl, S. T. Allen, M. Case, and M. J. W. Rodwell, "Millimeter-wave on-wafer waveform and network measurements using active probes," IEEE Trans. Microw. Theory Tech., vol. 43, no. 4, pp. 721-729, Apr. 1995.

[4] R. Y. Yu, J. Puls, K. Yoshiyuki, M. Case, K. Masayuki, and M. Rodwell, "A time-domain millimeter-wave vector network analyzer," IEEE Microw. Guided Wave Lett., vol. 2, no. 8, pp. 319-321, Aug. 1992.

[5] G. F. Ross, "Transmission and reception system for generating and receiving base-band pulse duration pulse signals without distortion for short base-band communication system," U.S. Patent 3,728,632, Apr. 17, 1973.
[6] M. J. Rodwell, S. T. Allen, R. Y. Yu, M. G. Case, U. Bhattacharya, M. Reddy, E. Carman, M. Kamegawa, Y. Konishi, J. Pusl, and R. Pullela, "Active and nonlinear wave propagation devices in ultrafast electronics and optoelectronics," Proc. IEEE, vol. 82, no. 7, pp. 1037-1059, Jul. 1994.

[7] J. R. Alday, "Narrow pulse generation by nonlinear transmission lines," Proc. IEEE, vol. 22, no. 6, p. 739, Jun. 1964.

[8] D. W. van der Weide, "Delta-doped Schottky diode nonlinear transmission lines for 480-fs, 3.5-V transients," Appl. Phy. Lett., vol. 65, no. 7, pp. 881-883, Aug. 1994.

[9] C. J. Chen, P. K. A. Wai, and C. R. Menyuk, "Soliton fiber ring laser," Opt. Lett., vol. 17, no. 6, pp. 417-419, Mar. 1992.

[10] W. Andress, D. S. Ricketts, X. Li, and D. Ham, "Passive and active control of regenerative standing and soliton waves," in Proc. IEEE Custom Integrated Circuits Conf. (CICC), 2006, pp. 29-36.

[11] D. S. Ricketts and D. Ham, "A chip-scale electrical soliton modelocked oscillator," in IEEE Int. Solid-State Circuits Conf. (ISSCC) Dig. Tech. Papers, 2006, pp. 432-433.

[12] A. C. Scott, F. Y. F. Chu, and D. W. McLaughlin, "The soliton: A new concept in applied science," Proc. IEEE, vol. 61, no. 10, pp. 1443-1483, Oct. 1973.

[13] P. G. Drazin and R. S. Johnson, Solitons: An Introduction. Cambridge, U.K.: Cambridge Univ. Press, 1989.

[14] M. Remoissenet, Waves Called Solitons: Concepts and Experiments. New York: Springer, 1999.

[15] E. Ott and R. N. Sudan, "Damping of solitary waves," The Physics of Fluids, vol. 13, no. 6, pp. 1432-1434, Jun. 1970.

[16] D. Ricketts, "The electrical soliton oscillator," Ph.D. dissertation, Harvard Univ., Cambridge, MA, 2006.

[17] D. Ham and A. Hajimiri, "Concepts and methods in optimization of integrated LC VCOs," IEEE J. Solid-State Circuits, vol. 36, no. 6, pp. 896-909, Jun. 2001.

[18] W. Andress and D. Ham, "Standing wave oscillators utilizing waveadaptive tapered transmission lines," IEEE J. Solid-State Circuits, vol. 40, no. 3, pp. 638-651, Mar. 2005.

[19] G. J. Ballantyne, P. T. Gough, and D. P. Taylor, "Periodic solutions of Toda lattice in loop nonlinear transmission line," Electron. Lett., vol. 29, no. 7, pp. 607-609, Apr. 1993.

[20] G. J. Ballantyne, "Periodically amplified soliton systems," Ph.D. dissertation, Univ. of Canterbury, Christchurch, New Zealand, 1994.

[21] H. A. Haus, "Mode-locking of lasers," IEEE J. Sel. Topics Quantum Electron., vol. 6, no. 6, pp. 1173-1185, Nov./Dec. 2000.

[22] C. C. Cutler, "The regenerative pulse generator," Proc. Inst. Radio Eng., vol. 43, no. 2, pp. 140-148, Feb. 1955.

[23] D. Ham, X. Li, S. A. Denenberg, T. H. Lee, and D. S. Ricketts, "Ordered and chaotic electrical solitons: communication perspectives," IEEE Commun. Mag., vol. 44, no. 12, pp. 126-135, Dec. 2006.

[24] E. Ott, Chaos in Dynamical Systems. Cambridge, U.K.: Cambridge Univ. Press, 1993.

[25] K. Cuomo and A. Oppenheim, "Synchronization of Lorenz-based chaotic circuits with applications to communications," IEEE Trans. Circuits Syst. II, Analog Digit. Signal Process., vol. 40, no. 10, pp. 626-633, Oct. 1993.

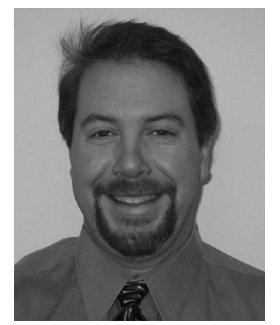

David S. Ricketts (S'98-M'06) received the B.S and M.S. degrees in electrical engineering from Worcester Polytechnic Institute, Worchester, MA, and the Ph.D. degree in electrical engineering from Harvard University, Cambridge, MA.

$\mathrm{He}$ is an Assistant Professor of Electrical and Computer Engineering at Carnegie Mellon University, Pittsburgh, PA. He worked in industry from 1995 to 2003 developing a wide array of analog and mixed-signal integrated circuits in BJT and submicron CMOS technologies. From 2003 to 2006, he was a research assistant at Harvard University developing soliton-based circuits and nanowire flexible electronics. His current research interests include ultra-fast RF integrated circuits and devices, soliton electronics, nanoscale electronics, and quantum devices.

Dr. Ricketts was awarded the Analog Devices Fellowship at Worcester Polytechnic Institute, was a 2004 Innovation Fellow at Harvard University, and was awarded the Analog Devices Outstanding Student Designer Award in 2006. 


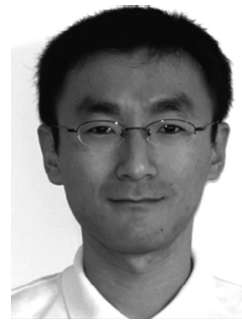

Xiaofeng Li (S'05) attended Tsinghua University, Beijing, China for undergraduate study in 1998 . After two years, he transferred to California Institute of Technology (Caltech), Pasadena, where he received the B.S. degree in electrical engineering in 2004. He is currently working toward the Ph.D. degree in electrical engineering and applied physics at Harvard University, Cambridge, MA.

His main research interest lies in the design and experimentation of gigahertz-to-terahertz ultrafast quantum circuits using low-dimensional nanoscale devices, such as quantum dots, nanowires, and carbon nanotubes.

$\mathrm{Mr}$. Li was the recipient of a Gold Medal at the 29th International Physics Olympiad, Reykjavik, Iceland, 1998. He ranked first in the Boston Area Undergraduate Physics Competition (U.S. intercollegiate physics competition) in both 2001 and 2002. He was also the recipient of the 2002 California Institute of Technology Henry Ford II Scholar Award, the 2004 Harvard University Pierce Fellowship, and the 2005 Analog Devices Outstanding Student Designer Award.

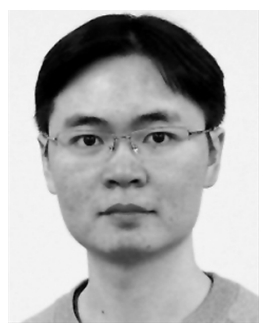

Nan Sun (S'06) received the B.S. degree in electrical engineering from Tsinghua University, Beijing, China, in 2006. He is currently working toward the Ph.D. degree in electrical engineering at Harvard University, Cambridge, MA, where his major research interests lie in RF, analog and mixed-signal integrated circuit design, and also in silicon bio-molecular sensors.

He ranked first in the Department of Microelectronics at Tsinghua University in graduation. He was the recipient of the Samsung Outstanding Student Award in 2003, Hewlett Packard Outstanding Student Award in 2006, and Analog Device Outstanding Student Designer Award in 2007.

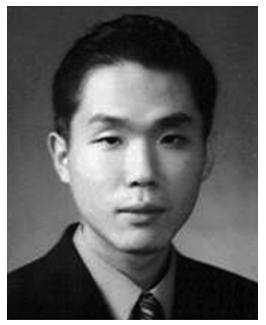

Kyoungho Woo (S'03) received the B.S. degree from Seoul National University, Seoul, Korea, in 1999, and the M.S. degree from Stanford University, Stanford, CA, in 2004, all in electrical engineering. He is currently working toward the Ph.D. degree in electrical engineering at Harvard University, Cambridge, MA, where his research focus is on PLL frequency synthesizers and high-speed serial links.

In 2003, he was with the Sun Microsystems, Sunnyvale, CA, where he developed a timing analysis code for the Ultrasparc III microprocessor. He is currently working as a part-time employee at Cavium Networks, Marlborough, MA, where he is developing a multi-standard high-speed serial links transmitter.

Mr. Woo is a Fellow of the Korea Foundation for Advanced Studies, and is the recipient of the Analog Devices Outstanding Student Designer Award in 2006.

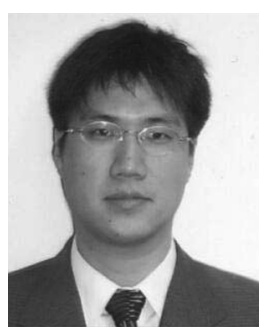

Donhee Ham (S'99-M'02) received the B.S. degree in physics in 1996 from Seoul National University, Korea, graduating with Presidential Honor atop the Natural Science College, and the Ph.D. degree in electrical engineering from the California Institute of Technology (Caltech), Pasadena, in 2002, winning the Charles Wilts Doctoral Thesis Prize (best thesis award in electrical engineering).

Dr. Ham is currently an Associate Professor in electrical engineering and applied physics with the School of Engineering and Applied Sciences, Harvard University, Cambridge, MA. His work experience also includes the Caltech-MIT Laser Interferometer Gravitational Wave Observatory (LIGO), 1997/1998, IBM T. J. Watson Research Center, 2000, IEEE conference technical program committees including International Solid-State Circuits Conference (ISSCC), and industry/government technical advisory positions on subjects including ultrafast solid-state electronics and science and technology at the nanoscale. He is a co-editor of CMOS Biotechnology (Springer, 2007). The current research focus of his research group at Harvard is on: RF, microwave, analog and mixed-signal ICs; gigahertz-to-terahertz ultrafast quantum circuits using low-dimensional nanoscale devices; soliton and nonlinear wave electronics; and applications of CMOS ICs in biotechnology.

Dr. Ham was the recipient of the Caltech Li Ming Scholarship and IBM Graduate Research Fellowship. He was also the recipient of the 2003 IBM Faculty Partnership Award. He shared Harvard's Hoopes prize (best senior thesis award) in 2003 with Mr. William Andress. 